\title{
Valid and Reliable Measure of Adherence to Satter Division of Responsibility in Feeding
}

\author{
Barbara Lohse, PhD, RDN, $\mathrm{CDN}^{1, \dagger}$; Diane C. Mitchell, MS, $\mathrm{RD}^{2}$
}

\begin{abstract}
Objective: To examine the validity and psychometrics of sDOR.2-6y, a 12-item measure of adherence to the Satter Division of Responsibility in Feeding (sDOR).

Design: Cross-sectional survey.

Setting: Online respondents in central Pennsylvania.

Participants: 117 parents (94\% female, 77\% White, 62\% in $\geq 1$ income-based assistance program) of preschoolers aged $2-6$ years (28\% moderate/high nutrition risk).

Main Outcome Measures: The sDOR.2-6y and Nutrition Screening Tool for Every Preschooler (NutriSTEP), a measure of child nutrition risk and other validated measures of eating behavior and parent feeding practices.

Analysis: Relationships were evaluated with Pearson $r, t$ tests, ANOVA, or chi-square. Factor structure was investigated using principal components analysis with varimax rotation. Binary logistic regression and general linear model controlling for low-income status compared with sDOR.2-6y and NutriSTEP scores. Linear regression predicted NutriSTEP and Satter Eating Competence Inventory 2.0 scores from sDOR.2-6y.

Results: The sDOR.2-6y ranged from 16-32 (mean, 25.9 $\pm 3.3 ; \mathrm{n}=114$ ). Parents of youth at nutrition risk had lower sDOR.2-6y scores $(P=0.004)$. Each 1 point sDOR.2-6y increase decreased nutrition risk odds by $21 \%$ (95\% confidence interval, $0.675-0.918 ; P=0.002)$. The sDOR.2-6y scores were higher with less restriction and pressure to eat (both $P<0.001$ ) and were associated with feeding style. Specificity was $87 \%$ with sDOR.2-6y cutoff $\geq 24$; sensitivity was $66 \%$ with cutoff $\geq 26$.

Conclusions and Implications: The sDOR.2-6y accurately and reliably indicated adherence of lowincome mothers to sDOR. Larger, diverse samples for future studies are recommended.

Key Words: parent feeding practices, child nutrition risk, early childhood health, construct validity, survey development (J Nutr Educ Behav. 2021;53:211-222.)

Accepted November 12, 2020. Published online January 8, 2021.
\end{abstract}

\section{INTRODUCTION}

In a position statement addressing nurturing healthy eating in children, 4 themes were identified that promote healthy feeding practices: positive parental feeding (eg, avoiding food restriction, letting children choose from food choices), eating together, a healthy home food environment, and pleasure of eating. ${ }^{1}$ The Satter Division of Responsibility in Feeding (sDOR) melds these 4 themes with parent agency for the feeding experience categorized as parent leadership and autonomy

\footnotetext{
${ }^{1}$ Wegmans School of Health and Nutrition, Rochester Institute of Technology, Rochester, NY

${ }^{2}$ Diet Assessment Center, Department of Nutritional Sciences, The Pennsylvania State University, University Park, PA

$\dagger$ Dr Lohse was affiliated with the Department of Nutritional Sciences, The Pennsylvania State University, at the time this study was conducted.

Conflict of Interest Disclosure: The authors have not stated any conflicts of interest.

Address for correspondence: Barbara Lohse, PhD, RDN, CDN, Wegmans School of Health and Nutrition, Rochester Institute of Technology, 180 Lomb Memorial Dr 78-A622, Rochester, NY 14623; E-mail: balihst@rit.edu

(C) 2020 The Authors. Published by Elsevier Inc. on behalf of Society for Nutrition Education and Behavior. This is an open access article under the CC BY-NC-ND license (http:// creativecommons.org/licenses/by-nc-nd/4.0/)

https://doi.org/10.1016/j.jneb.2020.11.007
}

support. As articulated in the writings and teachings of Ellyn Satter, ${ }^{2,3}$ sDOR posits that parents are responsible for what is presented to the child to eat, the emotional and physical environment (where), and timing (when). Furthermore, parents provide autonomy support, meaning that they trust children to determine what, how much, and whether they eat from what is served in the environment in which it is presented. As a trust model, sDOR is unique because child competence usurps the usual child deficit approach, meaning that sDOR does not assume that children will overeat, get fat, or focus only on less nutrientdense foods unless corralled into rules by well-intentioned parents. The sDOR is a theoretically grounded, research-supported ${ }^{4-13}$ approach that is used in medical, public health, and early childhood health education venues. $^{14-19}$ 
The application of sDOR has been hampered by the lack of an evaluation tool that specifically measures adherence to the entire approach. Many parent feeding tools include elements of sDOR, but only 1 , the sDOR.2-6y, addresses all the tenets of sDOR, ${ }^{20,21}$ and few have been rigorously tested for validity and reliability. ${ }^{21-23}$ The sDOR.2-6y is a 12 -item measure of sDOR adherence for parents of children aged at least 2 years but not past their sixth birthday that has undergone testing with cognitive interviews and observational measures to identify face and content ${ }^{24}$ and construct validity. ${ }^{25}$ These stages of development are consistent with recommendations for developing health behavior scales, ${ }^{23}$ including multi-staged processes that require time and patience. ${ }^{22}$ Response mapping of cognitive interviews with 5 separate samples reduced an early version of the sDOR.2-6y from 38 to 15 items, $^{24}$ and comparisons by sDOR experts of video-captured family meals with parent responses to the 15-item sDOR.2-6y supported content and predictive construct validity for 12 of the items. ${ }^{25}$ Subsequent methods in survey development and validation include testing for reliability (internal consistency reliability and test-retest reliability), criterion, and convergent or divergent construct validity. ${ }^{23}$ Criterion validation relates performance on 1 instrument to that on another validated measure; concurrent criterion validation is when both measures are completed simultaneously. Convergent construct validity denotes performance on an operational measure of a nonobservable trait (ie, a construct) that is similar to that of another measure of a theoretically related construct. Additional investigation is crucial for understanding instrument validity. Relying on only 1 or 2 sources of evidence for validity is "...not in line with current recommendations provided by the large scientific and professional associations in the psychological and educational fields ...". ${ }^{26}$ Messick's ${ }^{27}$ seminal treatise on validity clarifies that "Validity is not a property of the test or assessment as such, rather of the meaning of the test scores....Validity is an evolving property and validation is a continuing process." Therefore, the purpose of this study was to continue the examination of construct validity, specifically concurrent criterion and convergent validity of the sDOR.2-6y.

\section{METHODS}

\section{Study Design and Sample}

This study used a cross-sectional design to assess criterion and convergent construct validity of the SDOR.2$6 y$ with an online survey set including this and domain-related measures as well as 3 online 24-hour recalls to assess dietary quality. A subset of the sample completed a second survey administration to examine test-retest reliability. Inclusion criteria were being a Pennsylvania resident, being a primary caregiver of at least 1 child between 24 and 72 months who is not a ward of the state and without a disease diagnosis that could influence the child's intake, not working as or training to be a nutritionist, and having Internet access.

\section{Recruitment}

Participants were recruited through flyers and cards placed in public venues throughout central Pennsylvania (eg, YMCAs, public libraries, WIC offices, laundromats, free clinics). The sDOR.2-6y was available in English only, and hence, recruitment materials were in English. Recruitment materials described the study and included a link to the online survey. On accessing the link, to continue, participants responded to questions to affirm meeting the inclusion criteria. A paper survey option was available as an accommodation to those who expressed interest in the study but indicated having problems with Internet access.

A $\$ 10$ e-gift card was provided after survey submission; $\$ 10, \$ 15$, and $\$ 20$ e-gift cards were provided for completing the first, second, and third 24-hour dietary recalls, respectively. Recalls were completed over a period of $<1$ to 3 weeks and were from at least 1 weekday and 1 weekend day. The study was reviewed and approved by the Pennsylvania State University and Rochester Institute of Technology Institutional Review
Boards for the Protection of Human Subjects. For eligible respondents, informed consent was available online with an option to print the consent form. Separate consents were provided for the survey, diet assessment, and retest components of the study.

\section{Measurements}

To examine concurrent criterion validity, the authors compared sDOR.2-6y with validated and tested measures and items congruent with tenets of sDOR (eg, parental nonuse of restriction or pressure to manipulate intake, parent feeding style). In addition, decreased child nutrition risk and more healthful parent eating behaviors (eg, eating competence [EC] and lower emotional eating) are suggested outcomes of adherence to sDOR. Therefore, to examine convergent construct validity, the online survey set consisted of the sDOR.2-6y and 9 validated instruments, and demographic and food resource related items that could examine the performance of sDOR.2-6y in identifying these sDOR tenets. The order of instrument placement in the survey set was identical for all respondents; sDOR.2-6y was first in the survey set.

Satter Division of Responsibility. Adherence to sDOR was measured with the 12-item sDOR.2-6y. ${ }^{24,25}$ Each item had 5 response options (always, often, sometimes, rarely, never) that were scored from 3 to 0 . Six items were phrased so that a positive response indicated sDOR adherence; a disagreement with 5 items was interpreted as SDOR adherence; and 1 item denoted sDOR adherence as neither strong agreement nor disagreement, but rather a less frequent but apparent occurrence. To ensure congruence with sDOR elements, positively phrased items were assigned scores of 3 (always), 2 (often), 1 (sometimes), and 0 (rarely or never); reverse-scored items were assigned scores of 3 (rarely or never), 2 (sometimes), 1 (often), 0 (always); and responses to "I decide what foods to buy based on what my child eats" were assigned values of 3 (sometimes), 2 (often), 1 (rarely), and 0 (always, never). Possible scores were from 0 to 36 . 
Child nutrition risk. The validated and reliable Nutrition Screening Tool for Every Preschooler (NutriSTEP) ${ }^{28-30}$ assessed child nutrition risk. The 17 items, which address 4 risk factors (dietary intake, physical growth, physical activity, and influences on eating behaviors), have $2-5$ response options. Response options for an item each have an assigned value that is summed and categorized. Overall scores may range from 0 to 68 ; scores $\leq 20$ indicate no nutrition risk, scores 21-25 indicate moderate nutrition risk, scores $\geq 26$ indicate high nutrition risk. The NutriSTEP has been tested and found to be easy for parents to complete ${ }^{31}$ and reliable for online use. ${ }^{32}$ The sensitivity and specificity of sDOR.2-6y to identify child nutrition risk were established with NutriSTEP scores.

Quality of life. Child quality of life was measured with the Pediatric Quality of Life Inventory ${ }^{33}$ for toddlers (aged $2-4$ years). This validated instrument consists of 18 items with 5 response options summed to form 3 subscales: physical functioning (8 items), emotional functioning (5 items), and social functioning (5 items). Scores are transformed to percentiles with higher scores indicating higher quality of life for toddlers. Cronbach $\alpha$ in this sample was 0.87 .

Perceived stress. Parent perceived stress was assessed by a single item that is from the Community Health Database. ${ }^{34}$ The visual analog scale is anchored by 1 (no stress) and 10 (extreme stress).

Three-Factor Eating Questionnaire-R18 (TFEQ). Parent eating behavior was assessed with the validated 18-item TFEQ. ${ }^{35}$ This survey consists of 18 items, each with 4-point response options, grouped into 3 scales: cognitive restraint (6 items, possible score 6-24); uncontrolled eating (9 items, possible score 9-36); and emotional eating (3 items, possible score 3-12). For each scale, lower scores indicate less congruence with the eating behavior. Cronbach $\alpha$ in this sample was 0.75 for cognitive restraint, 0.71 for uncontrolled eating, and 0.78 for emotional eating.
Eating competence. Eating competence was measured with the 16-item validated and reliability-tested Satter Eating Competence Inventory (ecSI2.0). ${ }^{36,37}$ Each item has 5 response options assigned values ranging from 3 to 0 so that possible scores range from 0 to 48 , with higher numbers indicating greater EC. Scores $\geq 32$ indicate EC. The following 4 subscales align with the EC construct: eating attitudes, food acceptance, internal regulation, and contextual skills. Cronbach $\alpha$ in this sample was 0.89 .

Parent feeding styles. Parent feeding behaviors were categorized with the validated Caregivers Feeding Style Questionnaire. ${ }^{38}$ Each of the 19 items had 5 response options that were summed and averaged to determine levels of parent demandingness and responsiveness. Predetermined cutoffs for low-income samples were applied to identify parent feeding styles as indulgent, uninvolved, authoritarian, or authoritative.

Child feeding. The Child Feeding Questionnaire (CFQ) is a tested, 31item tool with 5 response options per parent feeding attitude and practices item. ${ }^{39}$ Responses form 7 scales: perceived responsibility, perceived parent weight, concern about child weight, restriction, pressure to eat, and monitoring. Scale scores are averaged so that each ranges from 1 (less frequent) to 5 (more frequent).

Sleep quality. Subjective sleep quality, and 6 sleep practice domains (eg, sleep latency, sleep duration, daytime dysfunction) were measured using the validated Pittsburgh Sleep Quality Index (PSQI). ${ }^{40}$ Scales were scored according to analysis directions and were then summed to generate a global index sleep score that can range from 0 to 21; a global score $\geq 5$ indicated poor quality sleep.

General health. The General Health Questionnaire (GHQ) assessed anxiety and mood-related symptomatology with 12 items scored on a 4-point Likert scale from 0 (not at all) to 3 (much more than usual). Possible scores of this tool range from 0 to 36 , with higher scores indicating greater problems with health, stress, and emotional problems. ${ }^{41} \mathrm{~A}$ bimodal scoring strategy for each of the 12 items has been suggested, with a threshold of 4 or higher indicating concerns with anxiety and mood disorders. Cronbach $\alpha$ for this sample is 0.89 .

Dietary intake. Dietary intake was assessed from a minimum of 2 out of 3 possible 24-hour recalls collected with the National Cancer Institute online Automated Self-Assessment 24-hour dietary assessment tool. ${ }^{42}$

\section{Data Collection}

Survey data were collected using the Qualtrics online survey platform (Provo, UT). On seeing research staff in the clinic, a few parents requested and completed paper surveys because of Internet access issues; their responses were entered into the Qualtrics platform and verified by research personnel. After completing the survey set, respondents indicating an interest in the dietary assessment were reported to the Pennsylvania State University Diet Assessment Center and then emailed instructions to access the automated self-assessment 24-hour dietary collection platform for the 3 24-hour recalls. The Diet Assessment Center managed the dietary data collection and analysis process. The 24 -hour recall requests were unannounced and included opportunities to report intake on weekends and weekdays. Weight and height were self-reported; a self-reported prepregnancy weight was used for pregnant or lactating women. To assess test-retest reliability, researchers emailed a survey link to complete the sDOR.2-6y a second time to respondents indicating interest in having an in-home meal captured by video. Pilot testing verified survey flow and online functionality.

\section{Data Analysis}

All surveys were scored according to published guidelines, and participant characteristics were reported using descriptive statistics. ${ }^{28,33-42}$ Lowincome status was identified as often or always worrying about money for 
food or participation in an incomebased assistance program. Nutrition Screening Tool for Every Preschooler scores were categorized using previously defined ranges ${ }^{28}$ into low, moderate, or high nutrition risk and grouped as low vs moderate/high risk. Tenets of EC were examined by comparing ecSI 2.0 scores to BMI, Healthy Eating Index (HEI), TFEQ, PSQI, GHQ, Pediatric Quality of Life Inventory, stress scale, and NutriSTEP responses. Associations between or among items from separate surveys were assessed with Pearson correlation or ANOVA as appropriate. Psychometrics of sDOR.2-6y were examined with Cronbach $\alpha$ to measure internal consistency and factor analysis using principal components analysis and varimax rotation to examine if items clustered by sDOR constructs.

To examine concurrent criterion and convergent construct validity, sDOR.2-6y scores were compared with NutriSTEP scores using binary logistic regression and univariate general linear models to control for low-income status. Linear regression was used to predict NutriSTEP and ecSI 2.0 scores. The NutriSTEP risk categories, sDOR.2-6y groupings and other survey categories (eg, parent feeding style or sleep quality), were compared with $t$ tests, 1-way ANOVA, or Pearson chi-square, as appropriate. $P<0.05$ was considered statistically significant. Significant ANOVA tests were followed by a Scheffe post hoc test to account for unequal group sizes. Chi-square values from contingency testing were examined post hoc with adjusted standardized-residuals. ${ }^{43,44}$ Significance levels from multiple comparisons were adjusted with the Bonferroni method. Sensitivity and specificity were calculated comparing sDOR.2$6 y$ scores against NutriSTEP risk categories. Dietary data from at least 2 recalls were averaged and analyzed using HEI 2010 guidelines.

A priori power calculations used pilot study findings with a mixedincome sample in the same geographic region. Pilot study sDOR.2$6 y$ mean was 26.2 , with an SD of 3.68. The sample size needed to detect a clinically significant difference of 3 points between 2 groups with a power of 0.9 was 60 (30 per group). To detect this difference between 4 groups with a power of 0.8 required a sample of 120 (30 per group). Observed power was reported with unequal group sizes. Assumptions of normality, homogeneity of variance, equality of errors variance, and multicollinearity were tested and met for all pertinent analyses. Data were analyzed with IBM SPSS Statistics for Macintosh (version 25.0; IBM Corporation, Armonk, NY, 2018)

\section{RESULTS}

Of the 175 clicks on the survey link, 117 submitted a survey (Figure), but the sample size ranged from 102 -117 because of missing responses for some items; the PSQI was completed by 82 . Of the 117 surveys, 109 were completed online, and 8 surveys used a paper format. Participants were mostly mothers (90\%; fathers 5\%; grandparents 4\%; significant other to parent $1 \%$ ), under the age of 35 years, White, with some post -high school training (Table 1). Ethnicity and race, which were asked as 2 separate questions, were self-reported by the children's parents. After selecting Hispanic or non-Hispanic, parents checked all that applied from a list, including American Indian/Alaskan Native, Asian, Black/African American, Native Hawaiian/other Pacific Islander, and White. At least $60 \%$ were overweight or obese; a majority used $\geq 1$ incomebased assistance programs. On average, parents reported a high level of stress as well as poor sleep quality. Using bimodal scoring, the GHQ indicated that $19 \%$ possibly had some issues with anxiety and mood disorders (score $\geq 4$ ). Two thirds of families included 2 or more children. More than one fourth of the referenced children were at some level of nutrition risk; however, the pediatric quality of life was above the 90th percentile. At least 2 24-hour recalls were completed by $53 \%(n=62)$ of the responders (Figure). Diet assessment participants reported a higher pediatric quality of life overall and in the areas of social and physical functioning. In addition, they reported a lower tendency to pressure their child to eat more food. Education and race were different between dietary responders and nonresponders, with $53 \%$ of the responders and only $27 \%$ of the nonresponders reporting a college degree or postgraduate training $(P=0.005)$. Of the White participants, $60 \%(\mathrm{n}=54)$ participated in the dietary assessment, compared with $17 \%(n=2)$ of the Black participants in the sample; no participation differences were noted by Hispanic ethnicity. Diet assessment attriters $(n=10)$ did not differ from completers $(n=62)$ in age; BMI; sDOR.2-6y, NutriStep, or ecSI 2.0 scores; parent feeding style; education level; Hispanic ethnicity; or lowincome. However, attriters included a greater proportion of Black participants $(40 \%)$ and fewer White participants $(3 \%)$ than completers $(6 \%$ and $87 \%$, respectively).

Reported tenets of EC relating ecSI 2.0 to weight, eating behaviors, stress, and sleep quality were affirmed (Table 2). Higher ecSI 2.0 scores were associated with lower BMIs and less emotional and uncontrolled eating, better quality sleep, less stress, higher pediatric quality of life, better general health, and higher dietary quality. Lower NutriSTEP scores, indicating less child nutrition risk, were significantly associated with higher EC $(r=0.29, P=0.004)$. In addition, ecSI 2.0 subscale comparisons supported EC concepts (Supplementary Table 1).

Comparisons of similarly worded items from differing surveys supported respondent consistency (ie, relative validity) across the survey set. (Supplementary Table 2) For example, responses to the item about worrying about having enough money for food was highly correlated with the NutriSTEP "I have difficulty buying food to feed my child because food is expensive" $(|r|=0.76$, $P<0.001, \mathrm{n}=109$ ) and with the number of income-based assistance programs $(|r|=0.28, P=0.003, \mathrm{n}=109)$.

\section{Psychometric Profile}

Of 117 completing the sDOR.2-6y 114 completed all 12 items. Scores on the sDOR.2-6y ranged from 16-32 (possible range $0-36$ ) with a median score of 26 and a mean score of $25.9 \pm 3.3$. Scores were normally distributed. Using principal components analysis 


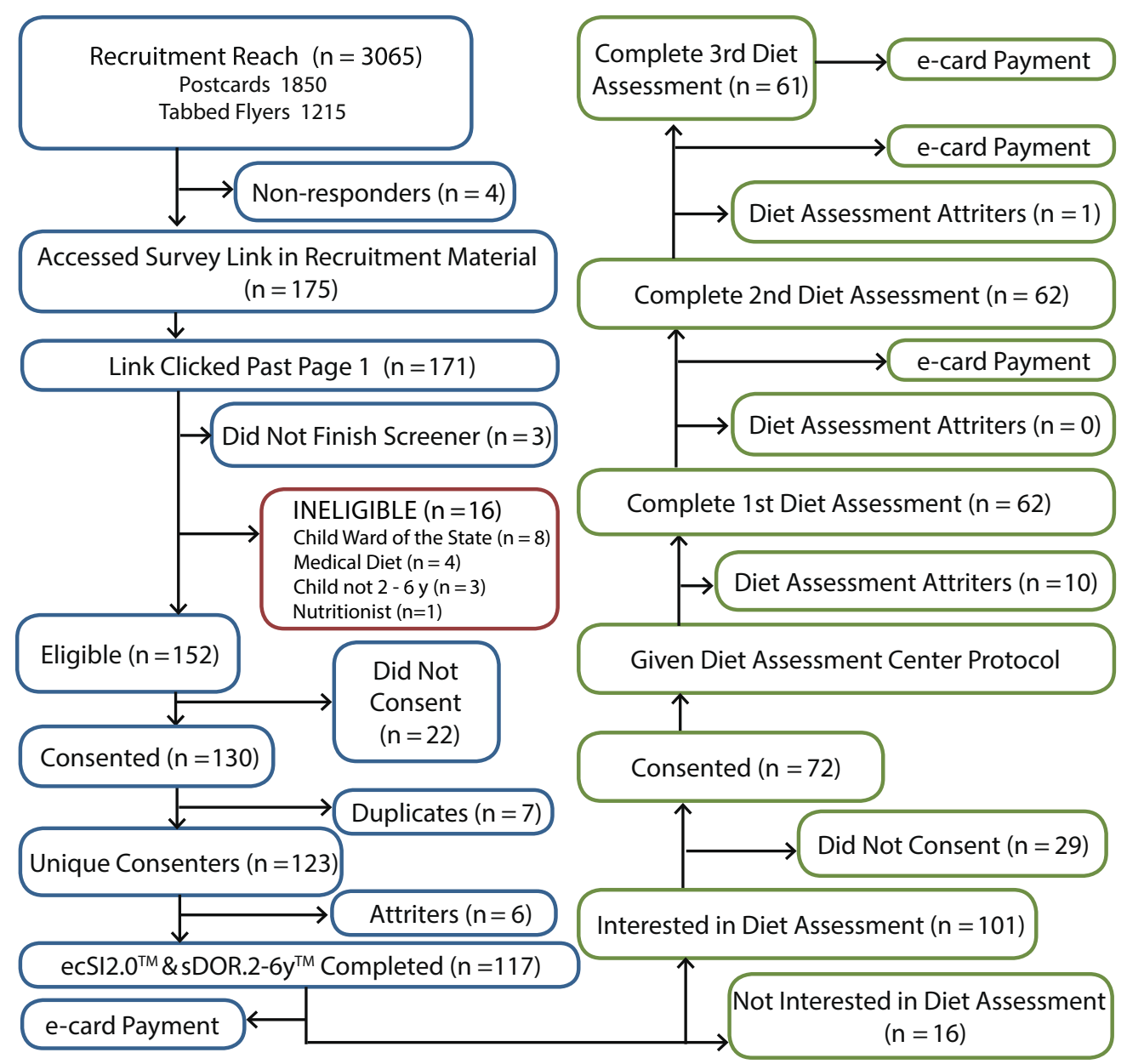

Figure. Participant recruitment, online access, eligibility, completion of the online survey, and participation in dietary assessment components to examine the validity of the sDOR.2-6y to measure adherence to the Satter Division of Responsibility in Feeding. Modified with permission from Lohse and Satter. ${ }^{25}$

with varimax rotation, researchers distributed the 12 survey items into 5 factors accounting for $59.2 \%$ of the variance. Factor loadings ranged from 0.47 to 0.81 , and each factor contributed evenly to the variance ranging from $12.9 \%, 12.6 \%, 11.6 \%, 11.4 \%$, and $10.8 \%$, respectively, for Factors $1-5$. The 5 factors described in Table 3 accurately depicted the theoretical underpinnings of sDOR, with 2 focused on child autonomy support concepts and 3 addressing parent leadership.

The 12 sDOR.2-6 items were not highly correlated. Only 9 of the possible 66 correlations had an absolute value greater than 0.2 , and the only correlation greater than 0.3 was between "My family has meals at about the same times every day" and "When I am home at mealtimes, I sit down and eat with my child" $(r=0.37)$, both of which factored on the mealtime structure factor (Table 3). Five of the 8 correlations with Pearson's $r$ between 0.20 and 0.30 involved the item: "I let my child have drinks (other than water) whenever s/he wants them." Cronbach $\alpha$ of the sDOR.2-6y was 0.32 (standardized 0.36) and ranged from $0.17-0.55$ for the 5 factors, which is congruent with the independence of the 12 sDOR.2-6 items. The sDOR.2$6 y$ was completed a second time by 18 parents $1-16$ weeks after the first administration; $50 \%$ repeated the survey within 4 weeks of the first completion. Repeat respondents, like the nonrepeat respondents, were also female $(90 \%)$, White $(95 \%, 5 \%$ Hispanic), with evidence of constrained food resources $(65 \%$ worried about money for food or use an incomebased assistance program). Repeat respondents were slightly older (35.3 \pm 6.4 years vs $31.5 \pm 7.9$ years, $P=0.05$ ) with more having a 4-year college or postgraduate education (70\% vs $35 \%, P=0.05)$. Time 1 scores $(26.8 \pm$ $3.4)$ were highly correlated $(r=0.71$, $P<0.001)$ with retest scores $(26.4 \pm$ 3.4). Test-retest means were not significantly different.

The Satter Division of Responsibility in Feeding approach considers both never and rarely as equal responses; however, because respondents had the option to select either, the psychometrics of items scored from 0 to 4 (ie, never and rarely having separate scores) were examined. The findings (eg, factor structure, interitem correlations, Cronbach $\alpha$ ) when items were scored $0-3$ were affirmed. 
Table 1. Characteristics of Total Sample, Diet Assessment, and Nondiet Assessment Participants

\section{Personal Characteristics}

Child age, y

Child sex-female

NutriSTEP scale ${ }^{\text {b }}$

Low risk

Moderate risk

High risk

PedsQL ${ }^{\text {,* }}$

Physical Functioning Scale ${ }^{* \star}$

Emotional Functioning Scale

Social Functioning Scale*

Parent age, y

Parent $\mathrm{BMI}^{\mathrm{d}}$

Parent BMI categories ${ }^{e}$

Underweight

Normal

Overweight

Obese

Parent sex-female

Parent ethnicity ${ }^{f}$

Hispanic

Asian

Black

American Indian/Alaskan Native

White

Parent education $^{\text {** }}$

Less than high school

High school/diploma

2-y college/training

4-y college degree

Postgraduate degree

Assistance program use

$\geq$ Assistance program

SNAP

WIC

Food pantry

TANF

Medical assistance

No. programs used

Worry about food money

Sometimes

Often/always

Low-income $^{9}$

Stress level ${ }^{\text {h }}$

Cognitive restraint ${ }^{i}$

Uncontrolled eating ${ }^{j}$

Emotional eating ${ }^{k}$

Eating competence'

Total ecSI 2.0 score

Eating competent

Child feeding style

Uninvolved

Indulgent

Authoritarian

Authoritative

Pregnant

Family size

1 child

$\geq 2$ children
All Participants

$3.4 \pm 1.0$

$66(60)$

$17.6 \pm 6.0$

$14.7 \pm 3.9$

$22.7 \pm 1.6$

$29.6 \pm 2.4$

$97.1 \pm 8.6$

$95.7 \pm 15.3$

$98.6 \pm 5.5$

$98.0 \pm 6.4$

$32.2 \pm 7.8$

$28.5 \pm 8.0$

110

110

110

110

4 (4)

40 (36)

28 (26)

38 (35)

103 (94)

9 (8)

5 (4)

12 (10)

3 (3)

$90(77)$

111

6 (5)

25 (23)

$34(31)$

$23(21)$

23 (21)

117

73 (62)

34 (29)

$47(40)$

13 (11)

$10(9)$

36 (31)

117

111

$1.7 \pm 1.9$

29 (26)

$14(13)$

111

102

107

109

111

114

114

78 (70)

$6.7 \pm 2.1$

$13.6 \pm 3.6$

$18.3 \pm 4.1$

$6.2 \pm 2.5$

$31.7 \pm 8.1$

58 (51)

22 (19)

42 (37)

26 (23)

24 (21)

103

111
62

62

62

62

62

62

62

62

62

62

62

59

$$
\text { (1) }
$$

62
62
62
62
62
62
62

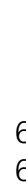

62

Diet Assessment

$\begin{array}{cc}3.5 \pm 1.0 & 55 \\ 33(53) & 48 \\ 16.8 \pm 5.6 & 41 \\ 46(78) & \\ 9(15) & \\ 4(7) & \end{array}$

$99.2 \pm 1.5 \quad 49$

$99.4 \pm 1.8 \quad 49$

$98.9 \pm 3.4 \quad 49$

$99.2 \pm 3.0 \quad 49$

$32.1 \pm 7.0 \quad 47$

$27.4 \pm 6.3 \quad 48$

62

62

62

$1.5 \pm 1.7$

62

62

61

60

62

$6.4 \pm 2.1$

$13.8 \pm 3.4$

$18.3 \pm 4.2$

$6.0 \pm 2.5$

61

61

62

$32.8 \pm 7.1$

59

62

34 (31)

77 (69)
2 (3)

24 (39)

$17(27)$

$19(31)$

59 (95)

5 (8)

3 (5)

2 (3)

$1(2)$

54 (87)

$1(2)$

$9(15)$

19 (31)

17 (27)

16 (26)

39 (63)

$16(26)$

25 (40)

5 (8)

1 (2)

20 (32)

$13(21)$

$8(13)$

43 (69)

34 (56)

$10(16)$

23 (37)

17 (27)

$12(19)$

No Diet Assessment

$3.3 \pm 1.0$

33 (69)

$18.7 \pm 6.5$

26 (63)

$10(24)$

5 (12)

$94.5 \pm 12.3$

$90.9 \pm 22.1$

$98.3 \pm 7.4$

$96.4 \pm 8.8$

$32.3 \pm 8.8$

$29.9 \pm 9.6$

2 (4)

16 (33)

11 (23)

$19(40)$

44 (92)

48

4 (8)

2 (4)

$10(18)$

2 (4)

36 (66)

49

$5(10)$

$16(33)$

15 (31)

$6(12)$

7 (14)

55

$34(62)$

18 (33)

$22(40)$

8 (15)

$9(16)$

16 (29)

$1.8 \pm 2.0$

49

16 (33)

$6(12)$

35 (71)

$7.2 \pm 2.1$

$13.4 \pm 3.9$

$18.3 \pm 3.9$

$6.5 \pm 2.4$

$30.4 \pm 9.0$

24 (45)

12 (23)

$19(37)$

$9(17)$

$12(22)$

6 (14)

15 (24)

19 (39)

30 (61) 
Table 1. (Continued)

\begin{tabular}{|c|c|c|c|c|c|c|}
\hline Personal Characteristics & $\mathbf{n}$ & All Participants & $\mathbf{n}$ & Diet Assessment & $\mathbf{n}$ & No Diet Assessment \\
\hline \multicolumn{7}{|l|}{$\mathrm{CFQ}^{\mathrm{m}}$} \\
\hline Perceived responsibility & 114 & $4.4 \pm 0.7$ & 62 & $4.4 \pm 0.6$ & 52 & $4.4 \pm 0.7$ \\
\hline Perceived parent wt & 112 & $3.2 \pm 0.4$ & 61 & $3.2 \pm 0.4$ & 51 & $3.2 \pm 0.5$ \\
\hline Perceived child wt & 114 & $2.9 \pm 0.3$ & 62 & $3.0 \pm 0.3$ & 52 & $2.9 \pm 0.2$ \\
\hline Concern child wt & 112 & $1.8 \pm 1.0$ & 61 & $1.8 \pm 1.1$ & 51 & $1.8 \pm 1.0$ \\
\hline Restriction & 112 & $3.4 \pm 0.8$ & 60 & $3.4 \pm 0.7$ & 52 & $3.5 \pm 0.9$ \\
\hline Pressure to eat* & 113 & $2.8 \pm 1.1$ & 61 & $2.6 \pm 1.1$ & 52 & $3.0 \pm 1.0$ \\
\hline Monitoring & 114 & $4.1 \pm 0.9$ & 62 & $4.2 \pm 0.8$ & 52 & $4.1 \pm 1.0$ \\
\hline$P S Q I^{n}$ & 82 & $6.3 \pm 3.7$ & 43 & $5.5 \pm 3.4$ & 39 & $7.3 \pm 3.9$ \\
\hline $\mathrm{GHQ}_{\text {total }}{ }^{\circ}$ & 111 & $11.4 \pm 5.5$ & 61 & $10.8 \pm 5.2$ & 50 & $12.2 \pm 5.9$ \\
\hline Psychological distress & 111 & $6.5 \pm 2.8$ & 61 & $6.2 \pm 2.4$ & 50 & $6.9 \pm 3.1$ \\
\hline Social dysfunction & 114 & $4.9 \pm 3.0$ & 62 & $4.7 \pm 3.0$ & 52 & $5.3 \pm 3.1$ \\
\hline \multicolumn{7}{|l|}{ Dietary information } \\
\hline Healthy Eating Index & & & 62 & $51.7 \pm 12.9$ & & \\
\hline Energy, cal & & & 62 & $1949 \pm 520$ & & \\
\hline Total protein, g & & & 62 & $77.7 \pm 21.5$ & & \\
\hline Total fat, g & & & 62 & $77.1 \pm 24.8$ & & \\
\hline Total sugar, g & & & 62 & $105.7 \pm 52.6$ & & \\
\hline
\end{tabular}

CFQ indicates Child Feeding Questionnaire; ecSI, Satter Eating Competence Inventory; GHQ, General Health Questionnaire; PedsQL, Pediatric Quality of Life Inventory; PSQI, Pittsburgh Sleep Quality Index; SNAP, Supplemental Nutrition Assistance Program; TANF, Temporary Assistance to Needy Families; WIC, Special Supplemental Nutrition Program for Women, Infants, and Children.

${ }^{a}$ Total participants $=117$; diet participants $=62$; numbers vary based on missing survey responses. Table entries are frequency (\%) except for mean \pm SD for child age; NutriStep scale scores; PedsQL; parent BMI, age, and stress level; cognitive restraint, uncontrolled eating, emotional eating scores; CFQ subscales; PSQI; GHI subscales; Dietary information: Healthy Eating Index, total protein, fat, and sugar; ${ }^{\mathrm{b}}$ NutriSTEP consists of 17 items with a possible score of 0-68. Risk categories: Low, $\leq 20$; Moderate, 21-25; High, $\geq 26$. The sample range was 4-33; median 17.5; ${ }^{\circ}$ PedsQL survey included 18 items; Physical functioning scale (8 items): Emotional Functioning Scale and Social Functioning Scale (5 items each). Scores were transformed to percentiles; higher scores indicate a higher quality of life; ${ }^{d}$ Parent BMI based on self-report height and nonpregnant weight; eUnderweight BMI <18.5; normal weight BMI 18.5-24.9; overweight BMI 25.0-29.9; Obese BMI $\geq 30$; ${ }^{\dagger}$ Some parents selected more than 1 ethnicity (\% of total sample). Values reflect the number selecting the specific choice; ' ${ }^{9}$ Low income defined as often

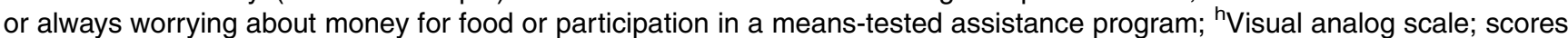
range from 1 (no stress) to 10 (extreme stress); 'The possible Three-Factor Eating Questionnaire-R18 Cognitive restraint scores are 6 (low) to 24 (high); 'The possible Three-Factor Eating Questionnaire-R18 Uncontrolled Eating scores are 9 (low) to 36 (high); ${ }^{\mathrm{T}}$ The possible Three-Factor Eating Questionnaire-R18 Emotional Eating scores are 3 (low) to 12 (high); 'ecSI 2.0 scores range from 0 (low) to 48 (high). Scores $\geq 32$ denote being eating competent; ${ }^{\mathrm{m}} \mathrm{CFQ}$ subscale scores range from $1-5$; higher scores reflecting greater feeding practice frequency; ${ }^{\mathrm{n}} \mathrm{PSQI}$ is a subjective measure of sleep quality consisting of 18 items. Possible scores range from 0-21. A global score of $\geq 5$ indicates a poor sleeper; ${ }^{\circ} \mathrm{GHQ}$ consists of 12 items with a possible score of 0 to 36. The Psychological Distress Scale consists of 7 items with a possible score of $0-21$, and the Social Dysfunction Scale consists of 5 items with a possible score of $0-15$; higher scores indicate worse health.

Note: Difference between diet and nondiet participants: ${ }^{*} P<0.05 ;{ }^{* *} P \leq 0.01$.

The sDOR.2-6y Compared With Measures of Health and Nutrition Risk in Children

Parents of low nutrition risk children $(n=70)$, based on NutriSTEP scores, reported more alignment with sDOR tenets than parents with youth at moderate or high nutrition risk $(\mathrm{n}=28)(26.7 \pm 2.9$ vs $24.3 \pm 3.5$; $\mathrm{t}=3.42, P=0.001)$. This relationship persisted even when controlling for low-income status $(26.9 \pm 0.4$ vs $24.3 \pm 0.6, P=0.001)$. The sDOR.2-6y scores were significantly different ( $F=5.80, P=0.004)$ when the 3 NutriSTEP risk categories were compared separately. Observed power when conducting these analyses was 0.9. Post hoc analyses revealed higher sDOR.2-6y scores between parents of low risk and moderate risk $(\mathrm{n}=19)$ children $(26.7 \pm 2.9$ vs $24.3 \pm 3.7$; $P=0.02$ ). Only 9 children were identified as high risk and their parents had sDOR.2-6y scores identical to moderate risk. sDOR.2-6y and NutriSTEP scores were significantly correlated $(r=-0.43, \quad P<0.001, \mathrm{n}=98)$ and sDOR.2-6y score predicted the NutriSTEP score $(\beta,-0.8 ; 95 \%$ confidence interval $[\mathrm{CI}],-1.14$ to -0.46 , constant 38.3; $P<0.001)$.

Binary logistic regression showed that for each 1-point increase in sDOR.2-6y, the odds of being in the NutriSTEP moderate or high nutrition risk category decreased by $21 \%$ (95\% CI, 0.675-0.918, $P=0.002$ ).

If sDOR adherence is designated as a score of $\geq 24$, sensitivity to detect moderate or high nutrition risk is 
Table 2. Comparison of Eating Competence Status With Selected Measures to Demonstrate Congruence With Eating Competence Tenets

Measure
BMI
Three-Factor Eating Questionnaire-cognitive restraint
Three-Factor Eating Questionnaire-emotional eating
Three-Factor Eating Questionnaire-uncontrolled eating
Pittsburgh Sleep Quality Inventory
Stress scale
General Health Questionnaire
Total score
Psychological distress scale
Social dysfunction scale
Pediatric Quality of Life Inventory
Total score
Social functioning scale
Emotional functioning scale
Physical functioning scale
NutriSTEP

$$
\begin{gathered}
\text { Eating Competent }^{a} \\
26.1 \pm 7.1, n=53 \\
13.7 \pm 3.6, n=53 \\
5.7 \pm 2.3, n=55 \\
17.1 \pm 3.8, n=54 \\
5.5 \pm 3.6, n=38 \\
6.1 \pm 2.2, n=49 \\
10.0 \pm 4.6, n=55 \\
5.8 \pm 2.1, n=55 \\
4.2 \pm 2.8, n=56 \\
n=54 \\
98.6 \pm 6.1 \\
98.6 \pm 6.2 \\
99.8 \pm 1.0 \\
97.8 \pm 10.0 \\
15.9 \pm 6.1, n=49
\end{gathered}
$$

Not Eating Competent

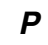

$\begin{array}{cl}30.1 \pm 7.7, n=54 & 0.008 \\ 13.3 \pm 3.5, n=52 & 0.51 \\ 6.8 \pm 2.5, n=54 & 0.02 \\ 19.5 \pm 4.0, n=53 & 0.002 \\ 7.0 \pm 3.7, n=43 & 0.09 \\ 7.2 \pm 2.0, n=50 & 0.01 \\ & \\ 12.7 \pm 6.0, n=53 & 0.01 \\ 7.2 \pm 3.2, n=53 & 0.01 \\ 5.5 \pm 3.0, n=55 & 0.02 \\ n=54 & \\ 95.6 \pm 10.5 & 0.07 \\ 97.2 \pm 6.7 & 0.27 \\ 97.4 \pm 7.7 & 0.03 \\ 93.4 \pm 19.3 & 0.14 \\ 19.1 \pm 5.6, n=50 & 0.007\end{array}$

BMI indicates body mass index; NS, not significant; NutriSTEP, Nutrition Screening Tool for Toddlers and Preschoolers. ${ }^{a}$ Eating Competence is defined as Satter Eating Competence Inventory 2.0 score $\geq 32$.

Note: Values are mean \pm SD.

\section{Table 3. Latent Variable Analysis of the sDOR.2-6y Using Principal Components Factor Analysis With Varimax Rotation}

\section{Factor $^{\text {b }}$}

1. Parent leadership with feeding: mealtime structure

2. Parent leadership with feeding: what is available to the child.

3. Parent leadership with feeding: how food is available to the child.

4. Child autonomy: respect for child autonomy in eating. ${ }^{d}$

5. Child autonomy: who controls what, when, or how much is eaten. ${ }^{c, d}$

\section{sDOR.2-6y Items}

My family has meals at about the same times every day. When I am home at mealtimes, I sit down and eat with my child.

I decide what foods to buy based on what my child eats.

I make something special for my child when s/he won't eat.

I let my child feed him/herself.

I am comfortable with providing meals for my family.

We have food leftover after meals.

If I think my child hasn't had enough, I try to get him or her to eat a few more bites.

I struggle to get my child to eat.

I let my child eat until s/he stops eating and doesn't want more.

I let my child eat whenever s/he feels like eating.

I let my child have drinks (other than water) whenever s/he wants them.
Factor Loading

0.770

0.789

0.809

0.708

0.727

0.472

0.479

0.607

0.602

0.650

0.774

0.533

sDOR indicates Satter Division of Responsibility.

${ }^{a}$ sDOR.2-6y A measure of adherence to the Satter Division of Responsibility; ${ }^{\text {s }}$ DOR.2-6y Cronbach $\alpha=0.32$. Factor-specific Cronbach $\alpha=0.55$ (Factor 1), 0.43 (Factor 2), 0.27 (Factor 3), 0.17 (Factor 4), and 0.19 (Factor 5); ${ }^{\circ}$ Correlation between 2 factors: $r=0.19, P=0.04, \mathrm{n}=114 ;{ }^{\mathrm{d}}$ Correlation between 2 factors: $r=0.23, P=0.01, \mathrm{n}=114$.

0.29 , and specificity is 0.87 . Sensitivity and specificity are 0.66 and 0.64 , respectively, if the sDOR adherence cutoff is adjusted to $\geq 26$. sDOR.2-6y scores were not related to the pediatric quality of life overall, social functioning, or physical functioning scores.
The sDOR.2-6y Compared With Measures of Health and Nutrition of Parents

Parents who had greater adherence to sDOR reported lower levels of stress $(r=0.21, P=0.02, \mathrm{n}=100)$. Higher Sleep quality (as measured by PSQI) was associated with greater adherence to sDOR $(r=0.33, P=0.003$, $\mathrm{n}=81)$. The sDOR.2-6y scores were significantly greater $(P=0.04)$ in parents with responses congruent with better sleep quality $(27.1 \pm 3.7, \mathrm{n}=$ 29) compared with poorer sleep quality $(25.5 \pm 3.2, \mathrm{n}=52)$. Observed 
power for comparing sDOR.2-6y scores between better and poorer sleep quality was 0.54 .

The sDOR.2-6y scores were significantly inversely related to cognitive restraint $(r=-0.19 ; P<0.05 ; \mathrm{n}=105)$. Uncontrolled eating and Emotional eating scales were not related to sDOR.2-6y scores or the risk category scores.

Parent adherence to sDOR was related to their EC $(\mathrm{r}=0.27 ; P=0.005$; $\mathrm{n}=111$ ). sDOR.2-6y scores were able to predict ecSI2.0 scores $(P=0.005)$ using the equation, $14.4+0.67$ (sDOR.2-6y value). Eating competence was higher in sDOR.2-6y categories associated with lower child nutrition risk, although the differences did not reach statistical significance $(32.6 \pm 7.2$ vs $29.4 \pm 10.0$ sDOR.2-6y scores $\geq 24$ vs $<24$, respectively, $P=0.07 ; 32.9 \pm 7.4$ vs $30.2 \pm$ 8.9 sDOR.2-6y scores $\geq 26$ vs $<26$, respectively; $P=0.09$ ).

Of the 12 sDOR.2-6y items, significant differences between parents with $(\mathrm{n}=21)$ and without $(\mathrm{n}=90)$ mood and anxiety concerns as denoted by the GHQ were noted for only 1 item: "My family has meals at about the same times every day" $(P=0.04)$. This sDOR.2-6y item was significantly correlated with 7 of 12 GHQ items (all $P \leq 0.02$ ). Items included those relevant to being able to have regular meals (eg, "Been able to concentrate on whatever you're doing, Felt constantly under strain, Been able to enjoy your normal dayto-day activities, and Been losing confidence in yourself"). Of 2 sDOR.2-6y items (ie, "I struggle to get my child to eat and I am comfortable with providing meals for my family"), considered a priori likely to be associated with some GHQ items, only the comparison between "I am comfortable with providing meals for my family" and the GHQ item, "Been feeling unhappy and depressed," was significant ( $r=$ $0.20, P=0.02, \mathrm{n}=114)$. Parent BMI was not related to sDOR.2-6y scores. The only significant correlation with parent Healthy Eating Indices was with NutriSTEP scores $(|r|=0.28, P=$ $0.03 ; \mathrm{n}=59$ ), which addresses child dietary quality. However, HEI and HEI components were not related to
sDOR.2-6y scores and did not differ among caregiver feeding styles.

\section{The sDOR.2-6 Compared With Measures of Parent Feeding Behavior: CFQ and Caregiver Feeding Styles Questionnaire}

Adherence to sDOR was associated with less use of restriction $(r=0.34, P$ $<0.001, \mathrm{n}=110)$ and lower pressure to eat $(r=0.47, P<0.001, \mathrm{n}=111)$ as assessed by the CFQ.

The sDOR.2-6y scores were significantly different according to parent feeding style $(P=0.03)$, with lower scores in the authoritarian parents $(\mathrm{n}=26)$ than in the uninvolved $(\mathrm{n}=21)$, indulgent $(\mathrm{n}=41)$, and authoritative $(\mathrm{n}=24)$ parents $(24.4 \pm$ $3.1,26.1 \pm 3.2,26.8 \pm 3.6,26.0 \pm$ 2.5 , respectively); observed power to detect sDOR.2-6y differences in parent feeding style was 0.70. Post hoc analyses revealed a significant difference between authoritarian and indulgent parents $(P=0.035)$. More parents using an authoritarian feeding style had an sDOR.2-6y score associated with a child being at nutrition risk when a score of 26 was used as the cutoff score (65\% vs $38 \%, 27 \%$, and $27 \%$ for authoritarian, uninvolved, indulgent, and authoritative, respectively; $P=0.02$ ).

\section{DISCUSSION}

This study revealed that sDOR.2-6y demonstrated concurrent criterion, convergent construct validity in a predominantly White sample of resource-constrained but educated parents because it could identify preschool age youth at nutrition risk as indicated by parent responses to the NutriSTEP survey.

\section{Psychometric Findings}

Although higher sDOR.2-6y scores indicated greater adherence to sDOR, identification of cutoffs for sDOR adherence is useful for screening purposes. Cutoffs were determined on the basis of the balance of sensitivity and specificity of sDOR.2-6y to detect youth, not in a low NutriSTEP risk category. Denoting sDOR adherence by a score of 24 or more has a specificity of $87 \%$, which means that only $13 \%$ who adhered to sDOR would be incorrectly labeled as nonadherent and identified for unnecessary intervention. A cutoff of 26 or more has a lower specificity of $64 \%$ but a higher sensitivity at $66 \%$, meaning that about one third of those who do not adhere to sDOR will not be identified. These levels of sensitivity and specificity are congruent with the NutriSTEP values of $53 \%$ and $79 \%$, respectively, ${ }^{28}$ and with other health behavior measures. $^{41,45}$ Messick $^{27}$ includes score interpretation or consequential aspect as a validity issue. Inherent in score interpretation are value implications, which can have social relevance. For example, changing values placed on child nutrition risk, parent feeding education, and sDOR concepts may suggest alternative cutoffs than those obtained from these sensitivity and specificity analyses.

Factor analysis identified 5 distinct but equal latent variables, unique from each other, descriptive of sDOR tenets, and in sync with the perspective that sDOR is multifaceted and complex. More specifically, a mosaic of parent feeding behaviors is necessary to define sDOR adherence. $^{2,3}$ The parsing of these items into 5 distinct factors and the limited correlation among the 12 sDOR.2-6y items are consistent with the low Cronbach $\alpha .{ }^{46}$ Critical examination of Cronbach $\alpha$ as a measure of reliability or internal consistency suggests that a low value does not delimit an instrument's usefulness or acceptance, but may highlight the heterogeneity of the construct being measured. ${ }^{46-49}$ Of the 12 items, 10 correlations were 0.31 or higher with the total score; 2 items correlated at 0.11 and 0.022. Removing these items either alone or together did not raise Cronbach $\alpha$ above 0.44 . Cortina $^{47}$ suggests an expected Cronbach $\alpha$ of 0.52 with 12 items and 3 dimensions when the item intercorrelation (similar to the 0.32 in this study) is 0.30; the sDOR.2-6y had 5 dimensions; thus, a lower Cronbach $\alpha$ would be expected. Taber ${ }^{48}$ suggests that there are limited grounds 
for adopting the heuristic that Cronbach $\alpha$ must be at least 0.70 and notes "...instruments with quite a low value of alpha can still prove useful in some circumstances" (eg, tests, diagnostic tools, and concept inventories), especially when studies of their agreement, calibration, or validity support their use.

\section{Comparisons With Measures of Child Nutrition Risk}

Similar to these respondents, the $\mathrm{Nu}$ triSTEP validation sample, which was Canadian, was mostly female and educated but had less evidence of constrained food resources. ${ }^{28}$ The NutriSTEP findings paralleled those of the validation samples. For example, in a sample of 269 parents, scores ranged from 4-46 with a median of $18,^{28}$ which compares to the range (4-33) and median (17.5) of this study. The NutriSTEP means for a much larger Canadian sample $(\mathrm{n}=1,076), 15.0 \pm 6.6^{50}$ and a trial using online administration $(\mathrm{n}=63){ }^{32}$ $17.7 \pm 6.9$, also corresponded with this study mean of $17.6 \pm 6.0$. Similarly, the risk category means of Simpson et $\mathrm{al}^{28}(16.1 \pm 5.7,21 \pm 6.4,28.5$ \pm 9.6 for low, medium, and high risk, respectively) mirrored those of this sample (Table 1).

The specificity of NutriSTEP to detect a clinically-derived nutrition risk was $0.69-0.79,{ }^{28}$ which reflected the specificity of sDOR.2-6y to detect moderate or high nutrition risk (as denoted by NutriSTEP) when the risk cutoff of sDOR nonadherence is $<24$. In addition, the sensitivity of NutriSTEP to detect a clinically-derived nutrition risk $(0.53-0.69)^{28}$ was similar to the sensitivity of the sDOR.2-6y to detect NutriSTEP moderate or high nutrition risk when the risk cutoff was $<26$. Thus, inadvertent, costly, or emotionally disturbing labeling of healthy preschoolers as being at nutrition risk can be minimized without neglecting to identify at least half of them who are at nutrition risk. The administration of NutriSTEP to Canadian preschool parents who were educated, mostly White, and mothers $(\mathrm{n}=437)$ identified nutrition risk as high for $7 \%$ of youth, moderate for $19 \%$ and low in $74 \%,{ }^{51}$ aligning with the distribution within risk categories of the smaller sample of American parents in this study (Table 1 ).

\section{Comparisons With Measures of Parent Health and Nutrition}

Although unhealthy feeding practices have been associated with parent psychological distress, ${ }^{52}$ GHQ total and subscale scores were not related to sDOR.2-6y scores. The usefulness of the GHQ as a screener for psychiatric morbidity related to anxiety and mood disorders has been challenged by some reports of item response bias and measurement error related to dimensionality. ${ }^{1,53-55}$ However, the GHQ individual item correlations and less frequent mealtime regularity among parents with psychiatric distress suggest that adherence to sDOR may be related to caregiver emotional health. Further examination, using additional tested measures of caregiver emotional health, is supported to determine its potential as a confounder in future sDOR.2-6y validation studies. ${ }^{52}$

Healthy Eating Index and HEI component scores were not related to sDOR.2-6y responses. Although the impact on and relation of parent feeding practices and styles with child dietary intake has been extensively studied, ${ }^{1}$ comparison of parent dietary intake and their parent feeding styles and practice are not apparent. In addition to the possibility that parent HEI scores may not equate with parent feeding behaviors, the small sample size may have contributed to the inability to observe any HEI and sDOR.2-6y association.

The strong relationship between NutriSTEP and sDOR.2-6y scores suggested that parent adherence to sDOR is related to lower child nutrition risk. The sDOR.2-6y scores were associated with greater caregiver EC and less cognitive restraint in eating and caregiver feeding style that was less authoritarian, less restrictive, and less pressuring, all-important sDOR tenets, ${ }^{56}$ congruent with other studies of caregiver feeding styles and mealtime practices. ${ }^{57}$ These eating and feeding associations supported the criterion validity of sDOR.2-6y to measure adherence to sDOR. Instituting sDOR enhances family lifestyles $^{58}$; lifestyle qualities of better sleep and fewer feelings of stress were associated with higher sDOR.2-6y scores.

Important strengths of this study were that the sDOR.2-6y items were previously tested for comprehension and were congruent with observed parent behaviors. The use of multiple validated surveys facilitated a more circumspect measure of how child health, parent emotional health, feeding practices, and dietary intake relate to sDOR.2-6y responses. Study conclusions must consider several limitations. Recruitment was from programs and venues serving low-income persons resulting in a predominantly resource-constrained sample. Cross context equivalence of findings was limited because the sample also lacked ethnic and racial diversity. The generalization of test-retest results is limited by the small sample size. However, the results from the ecSI 2.0 and NutriSTEP paralleled those previously reported with general samples, tempering this limitation. ${ }^{28,31,36,37}$ Surveys were all self-reported and completed onlinefactors that could compromise data integrity. However, IP addresses and emails were monitored, and follow-up emails were used to investigate identity (eg, asking height, weight, child age) before sending online payments. In addition, 101 of 117 respondents expressed interest in the dietary assessment, an activity requiring additional interaction with study personnel and the opportunity to identify a duplicate respondent; such an action was not likely to be completed by an imposter.

Furthermore, congruence between similar items from different surveys throughout the survey set (Supplementary Table 2) supported relative validity. Child weight was neither reported nor assessed, limiting its use as a descriptor or statistical control. The sDOR.2-6y administration assumed English literacy. Although the ability to read and understand English was not tested, recruitment was not from sites that encounter many non-English speaking clients. Future studies with a Spanish version of the sDOR.2-6y will be required to determine validation in samples that primarily speak Spanish. This sample consisted of children without serious health concerns, therefore, results cannot be extended to parents of 
youth living with serious health concerns without further study.

\section{IMPLICATIONS FOR RESEARCH AND PRACTICE}

The 12-item sDOR.2-6y, like NutriSTEP, can indicate child nutrition risk but has the benefit of being able to convey parent feeding behaviors in greater detail, making it useful for caregiver education and counseling, in addition to screening. Additional studies of sDOR.2-6y validity will benefit from larger and more diverse samples that include measured child weight and height and more robust measures of parent depression and emotional stability to assess generalizability. In addition, comparison of findings in children aged 2-3 years with 4 - and 5year-olds may lead to more targeted application and knowledge of sDOR. Future studies are suggested that incorporate clinical examination and health record data to substitute for reliance on self-report to affirm the status of nutrition risk screening tools.

\section{ACKNOWLEDGMENTS}

The work in this manuscript was funded by the USDA, Food and Nutrition Services Supplemental Nutrition Assistance Program Education program through an agreement between The Pennsylvania Department of Human Services and the Pennsylvania Nutrition Education TRACKS, The Pennsylvania State University.

The authors acknowledge the staff of The Pennsylvania State University Diet Assessment Center for assistance with dietary data collection and preparation, Kathryn Faulring, MPH, CHES, for her assistance with manuscript preparation, and Ellyn Satter, MS, for her contributions to the research. Some descriptive components of this study were presented in the 2015 annual meeting of the Society for Nutrition Education and Behavior in Pittsburgh, PA.

\section{SUPPLEMENTARY DATA}

Supplementary data related to this article can be found at https://doi. org/10.1016/j.jneb.2020.11.007.

\section{REFERENCES}

1. Haines J, Haycraft E, Lytle L, et al Nurturing children's healthy eating: position statement. Appetite. 2019; 137:124-133.

2. Ellyn Satter Institute. Raise a healthy child who is a joy to feed. https:// www.ellynsatterinstitute.org/how-tofeed/the-division-of-responsibility-infeeding/. Accessed November 6, 2020.

3. Satter E. The feeding relationship: problems and interventions. I Pediatr. 1990;117:S181-S189

4. Morris SE, Klein MD. Pre-feeding Skills: A Comprehensive Resource for Mealtime Development. Austin, TX: Pro-Ed; 2000.

5. Greenspan S, Lourie RS. Developmental structuralist approach to the classification of adaptive and pathologic personality organizations: infancy and early childhood. Am J Psychiatry. 1981; 138:725-735.

6. Birch LL, Fisher JA. Appetite and eating behavior in children. Pediatr Clin North Am. 1995;42:931-953.

7. Orrell-Valente JK, Hill LG, Brechwald WA, Dodge KA, Pettit GS, Bates JE. "Just three more bites": an observational analysis of parents' socialization of children's eating at mealtime. Appetite. 2007;48:37-45.

8. Rhee KE, Lumeng JC, Appugliese DP, Kaciroti N, Bradley RH. Parenting styles and overweight status in first grade. Pediatrics. 2006;117:2047-2054.

9. Kakinami L, Barnett TA, Séguin L, Paradis G. Parenting style and obesity risk in children. Prev Med. 2015;75:18-22.

10. Kröller K, Warschburger P. Associations between maternal feeding style and food intake of children with a higher risk for overweight. Appetite. 2008; 51:166-172.

11. Marty L, Chambaron S, Nicklaus S, Monnery-Patris S. Learned pleasure from eating: an opportunity to promote healthy eating in children? Appetite. 2018;120:265-274.

12. Shim JE, Kim J, Lee Y, STRONG Kids Team. Fruit and vegetable intakes of preschool children are associated with feeding practices facilitating internalization of extrinsic motivation. J Nutr Educ Behav. 2016;48:311-317.e1.

13. Kasper N, Ball SC, Halverson K, et al. Deconstructing the family meal: are characteristics of the mealtime environment associated with the healthfulness of meals served? J Acad Nutr Diet. 2019;119:1296-1304
14. US Department of Agriculture, Food and Nutrition Service. Child feeding messages, tips, advice and tools. https://www.fns.usda.gov/core-nutrition/child-feeding. Accessed November 5,2020 .

15. Barlow SE, Expert Committee. Expert committee recommendations regarding the prevention, assessment, and treatment of child and adolescent overweight and obesity: summary report. Pediatrics. 2007;120(suppl 4):S164-S192.

16. Butte N, Cobb K, Dwyer J, et al. The start healthy feeding guidelines for infants and toddlers. J Am Diet Assoc. 2004;104:422-454.

17. Ogata BN, Hayes D. Position of the Academy of Nutrition and Dietetics: nutrition guidance for healthy children ages 2 to 11 years. J Acad Nutr Diet. 2014;114:1257-1276.

18. Devall E. Promoting healthy feeding relationships and nurturing parenting practices. New Mexico WIC program. Washington, DC: US Department of Agriculture; 2006. https://wicworks.fns.usda.gov/ wicworks/Sharing_Center/spg/NM_ report.pdf. Accessed November 6, 2020.

19. Institute of Medicine. Framework for Dietary Risk Assessment in the WIC Program: Interim Report. Washington, DC: The National Academies Press; 2000.

20. Sanchez K, Spittle AJ, Allinson L, Morgan A. Parent questionnaires measuring feeding disorders in preschool children: a systematic review. Dev Med Child Neurol. 2015;57:798-807.

21. Heller RL, Mobley AR. Instruments assessing parental responsive feeding in children ages birth to 5 years: a systematic review. Appetite. 2019;138:23-51.

22. Vaughn AE, Tabak RG, Bryant MJ, Ward DS. Measuring parent food practices: a systematic review of existing measures and examination of instruments. Int J Behav Nutr Phys Act. 2013;10:61.

23. Boateng GO, Neilands TB, Frongillo EA, Melgar-Quiñonez HR, Young SL. Best practices for developing and validating scales for health, social, and behavioral research: a primer. Front Public Health. 2018;6:149.

24. Lohse B, Satter E, Arnold K. Development of a tool to assess adherence to a model of the division of responsibility in feeding young children: using response mapping to capacitate validation measures. Child Obes. 2014;10:153-168.

25. Lohse B, Satter E. Use of an observational comparative strategy demonstrated 
construct validity of a measure to assess adherence to the Satter Division of Responsibility in Feeding [published online ahead of print]. J Acad Nutr Diet. https://doi.org/10.1016/j.jand.2020.11. 008.

26. Gadermann AM, Guhn M, Zumbo BD. Estimating ordinal reliability for Likert-type and ordinal item response data: a conceptual, empirical, and practical guide. Pract Assess Res Eval. 2012;17:1-13.

27. Messick S. Validity of psychological assessment: validation of inferences from persons' responses and performances as scientific inquiry into score meaning. Am Psychol. 1995;50:741-749.

28. Randall Simpson JA, Keller HH, Rysdate LA, Beyers JE. Nutrition Screening Tool for Every Preschooler (NutriSTEP): validation and test-retest reliability of a parent-administered questionnaire assessing nutrition risk of preschoolers. Eur J Clin Nutr. 2008;62:770-780.

29. Randall Simpson JA, Gumbley J, Whyte K, et al. Development, reliability, and validity testing of Toddler $\mathrm{Nu}-$ triSTEP: a nutrition risk screening questionnaire for children 18-35 months of age. Appl Physiol Nutr Metab. 2015;40:877-886.

30. NutriSTEP. Nutrition screening tool for toddlers and preschoolers. http:// www.nutristep.ca/. Accessed November 6, 2020 .

31. Watson-Jarvis K, McNeil D, Fenton TR, Campbell K. Implementing the Nutrition Screening Tool for Every Preschooler (NutriSTEP ${ }^{\circledR}$ ) in community health centres. Can J Diet Pract Res. 2011;72:96-98.

32. Carducci B, Reesor M, Haresign H, et al. NutriSTEP ${ }^{\circledR}$ is reliable for internet and onscreen use. Can J Diet Pract Res. 2015;76:9-14.

33. Varni JW, Seid M, Rode CA. The PedsQL: measurement model for the pediatric quality of life inventory. Med Care. 1999;37:126-139.

34. Parks EP, Kumanyika S, Moore RH, Stettler N, Wrotniak BH, Kazak A. Influence of stress in parents on child obesity and related behaviors. Pediatrics. 2012;130:e1096-e1104.

35. Karlsson J, Persson LO, Sjöström L, Sullivan M. Psychometric properties and factor structure of the Three-Factor Eating Questionnaire (TFEQ) in obese men and women. Results from the Swedish Obese Subjects (SOS) study. Int J Obes Relat Metab Disord. 2000;24:1715-1725.

36. Godleski S, Lohse B, Krall JS. Satter Eating Competence Inventory subscale restructure after confirmatory factor analysis. J Nutr Educ Behav. 2019;51: 1003-1010.

37. Stotts JL, Lohse B. Reliability of the ecSatter Inventory as a tool to measure eating competence. J Nutr Educ Behav. 2007;39(5 suppl):S167-S170.

38. Hughes SO, Cross MB, Hennessy E, Tovar A, Economos C, Power TB. Caregiver's Feeding Styles Questionnaire. Establishing cutoff points. Appetite. 2012;58:393-395.

39. Birch LL, Fisher JO, Grimm-Thomas K, Markey CN, Sawyer R, Johnson SL. Confirmatory factor analysis of the Child Feeding Questionnaire: a measure of parental attitudes, beliefs and practices about child feeding and obesity proneness. Appetite. 2001;36:201-210.

40. Buysee D, Reynolds 3rd CF, Monk TH, Berman SR, Kupfer DJ. The Pittsburgh Sleep Quality Index: a new instrument for psychiatric practice and research. Psychiatr Res. 1989;28:193213.

41. Mann RE, Cheung JTW, Ialomiteanu A, et al. Estimating prevalence of anxiety and mood disorder in survey data using the GHQ12: exploration of threshold values. Eur J Psychiat. 2011; 25:81-91.

42. National Cancer Institute. Automated Self-Administered 24-Hour dietary assessment tool. https://epi.grants.cancer.gov/ asa24/. Accessed November 6, 2020.

43. Beasley TM, Schumacker RE. Multiple regression approach to analyzing contingency tables: post hoc and planned comparison procedures. J Exp Educ. 1995;64:79-93.

44. García-Pérez MA, Núñez-Antón V. Cellwise residual analysis in two-way contingency tables. Educ Psychol Meas. 2003;63:825-839.

45. Hess CR, Landa RJ. Predictive and concurrent validity of parent concern about young children at risk for autism. J Autism Dev Disord. 2012;42:575-584.

46. Tavakol M, Dennick R. Making sense of Cronbach's alpha. Int J Med Educ. 2011;2:53-55.

47. Cortina JM. What is coefficient alpha? An examination of theory and applications. J Appl Psychol. 1993;78:98-104.
48. Taber KS. The use of Cronbach's alpha when developing and reporting research instruments in science education. Res Sci Educ. 2018;48:1273-1296.

49. Vehkalahti K, Puntanen S, Tarkkonen L. Implications of dimensionality on measurement reliability. In: Schipp B, Krämer W, eds. Statistical Inference, Econometric Analysis and Matrix Algebra: Festschrift in Honour of Götz Trenkler. Heidelberg, Germany: Physica-Verlag Heidelberg; 2009:143-160.

50. Persaud N, Maguire JL, Lebovic G, et al. Association between serum cholesterol and eating behaviours during early childhood: a cross-sectional study. CMAJ. 2013;185:e531-e536.

51. Watson-Jarvis K, Fenton TR, McNeil D, Campbell K. Preschool nutrition risk in Calgary. Can J Diet Pract Res. 2011;72:e101-e106.

52. Jang M, Brandon D, Vorderstrasse A. Relationships among parental psychological distress, parental feeding practices, child diet, and child body mass index. Nurs Res. 2019;68:296-306.

53. Donath $\mathrm{S}$. The validity of the 12-item General Health Questionnaire in Australia: a comparison between three scoring methods. Aust N Z J Psychiatry. 2001;35:231-235.

54. Hankins M. The reliability of the twelve-item General Health Questionnaire (GHQ-12) under realistic assumptions. BMC Public Health. 2008;8:355.

55. Gnambs T, Staufenbiel T. The structure of the General Health Questionnaire (GHQ-12): two meta-analytic factor analyses. Health Psychol Rev. 2018; 12:179-194.

56. Davies WH, Satter E, Berlin KS, et al. Reconceptualizing feeding and feeding disorders in interpersonal context: the case for a relational disorder. J Fam Psychol. 2006;20:409-417.

57. Lopez NV, Schembre S, Belcher BR, et al. Parenting styles, food-related parenting practices, and children's healthy eating: a mediation analysis to examine relationships between parenting and child diet. Appetite. 2018;128:205-213.

58. Satter E. The Satter Feeding Dynamics Model of child overweight definition, prevention and intervention. In: O'Donahue W, Moore BA, Scott B, eds. Pediatric and Adolescent Obesity Treatment: A Comprehensive Handbook, New York, NY: Taylor and Francis; 2007:287-314. 